\title{
Gesundheitsprofil von Hausfrauen der Nordschweiz
}

\author{
Kurt Biener, Lison Roschewski, Meinrad Schăr
}

Institut für Sozial und Präventivmedizin der Universität zürich

\section{Einleitung}

Nach Abschluss der Untersuchungen an 1260 berufstätigen Männern sowie an 1036 berufstätigen Frauen aus 44 Stichprobenbetrieben der Nordschweiz werden die Ehefrauen der damals erfassten Männer in einer zusatzstudie untersucht.

\section{Material}

Insgesamt werden 400 Hausfrauen aufgeboten (Nationalfondsprojekt Nr. 6.186.076). Ziel der Arbeit ist es, die Parameter der berufstätigen und nicht-berufstätigen Probandinnen $z u$ vergleichen una auch mit den Ergebnissen der Männerstudie zu korrelieren.

\section{Ergebnisse}

Die vorläufige Auswertung der bisher 200 Fragebögen ergab folgende aufschlussreiche Hinweise; die Aussagen gelten vorbehaltlich der endqültigen Computerauswertung. Anamnestisch hatten 218 dieser Frauen irgendeine Krebserkrankung eines Familienmitgliedes angegeben. Verschiedenartige Operationen hatten über die Hälfte dieser Frauen hinter sich, z.B. Appendektomie 98, Tonsillo- bzw. Tonsillektomie 15\%, sonstige Eingriffe $32 \%$. Einen Unfali hatte bisher jede aritte durchgemacht. Gegenwärtige gesundheitliche störungen mit Arztbehandlung gaben $22 \%$ an, vergleichsweise bei den berufstätigen Frauen etwas mehr, bei den berufstätigen Männern etwas weniger. Innerhalb des gynäkologischen Profils wurden $12 \%$ Curettagen, $12 \%$ Unterbindungen, 88 Totalexstixpationen, 38 Extrauteringraviaitäten, $3 \%$ ovarektomien und 28 Mamma amputationen registriert. Insgesamt hatten sich 318 aller dieser Frauen bisher einem gynäkologischen Eingriff unterziehen müssen. zum zeitpunkt unserer Untersuchung nahmen nux $10 z$ dex Hausfrauen gegenuber 308 der berufstätigen Frauen im fertilen Alter die Antibabypille zu sich. 35\% dieser hausfrauen richten sich nach der Regel von Knaus Ogino, 68 benutzen die spirale. In $30 \%$ wird die Kontrazeption von den Ehemännern verantwortet (u.a. 138 Vasektomien).

Ein Uebergewicht von mehr als $10 \mathrm{~kg}$ laut Broca-Index wiesen 148 dex Frauen auf (berufstätige Frauen (28). Einen pathologischen Blutdruckwert von ibber $160 \mathrm{mmHg}$ systolisch und/ oder 95 rumg diastolisch wiesen 208 auf (berufstätige Frauen 12\%). 198 der Probandinnen erfüllten den Ergometertest mit vorgegebener Wattbelastung von 2 Watt $/ \mathrm{kg}$ Körpergewi.cht übex 6 Minuten nicht; die eine Hälfte dieser Fäile konnte aus krankheits- oder verletzungsbedingten Grinden den Test nicht durchführen, die andere Halfte jedoch musste den Test aus Konditionsmangel oder infolge einer Kreislauferkrankung aufgeben. Innerhalb des Freizeitverhaltens fiel auf, dass $23 \%$ dieser Hausfrauen von 20 bis 42 Jahren Sportklubmitglieder waren (berufstätige Frauen $27 \%$, berufstatige Männer 368 ). Nur 68 aller Hausfrauen rauchten bis 20 zigaretten am Tag, $5 \%$ rauchten 20 zigaretten und mehr; es waren nur 118 Raucherinnen unter diesen Probandinnen, bei den berufstätigen
Frauen waren es 298 und bei den berufstätigen Mănnern 48\%. Zuweilen zu Schmerzmitteln griffen 438 der Hausfrauen (bexufstätige Frauen jedoch in $65 \%$ und berufstätige Männer in 408 ).

\section{Diskussion}

Die Studie wird zahlreiche weitere Hinweise geben und wertvolle Vergleichsmöglichkeiten eröffnen. Zusätzliche Erhebungen innerhalb dieser studie betreffen u.a. die Bekleidungs- und Körperhygiene, die Freizeitgestaltung, die Ernährungsgewohnheiten, sowie Wohnungs- und Familienprobleme. Auffällig ist, dass über die Hälfte aller Hausfrauen einer stundenweisen oder halbtägigen Zusatzbeschäftigung nachgehen. Die Untersuchungen der insgesamt 400 Probandinnen werden kontinuierlich fortgeführt.

\section{Résumé}

Deux cents mënagères ont été examinées. $22 \%$ souffraient d'un mal quelconque et etaient en traitement medical, 11 z seulement fumaient. $43 \%$ utilisaient, occasionnellement, des angesigues.

\section{Summary}

Out of 200 housewives, $22 \%$ were under medical treatment at the time of our interview. only 118 were smokers. 438 occasionaly took medications against pain, e.g. headache.

\section{Itteratur}

(1) BIENER K., FOREST F., SCHAER M.: NOYMOgrammstudie. Repräsentativerhebung iber den Gesundheitszustand männlicher Betriebsangehöriger in der Nordschweiz. I. Mitteilung: Anamnesen und Gesundheitsverhalten. Schweiz. med. Wschr. 106, 1279 (1976).

(2) BIENER K., FOREST F., SCHAER M.: NOrmogrammstudie. Repräsentativerhebung uber den Gesundheitszustand männlicher Betriebsangehöriger in der Nordschweiz. II. Mitteilung: Physiologische und biochemische Befunde. Schweiz. med. Wschr. 106, 1413 (1976).

(3) BIENER K., FOREST F., SCHAER M., SCHWARZENBACH H.: Normogrammstudie. Repräsentativerhebung über den Gesundheitszustand männlicher Betrlebsangehöriger in der Nordschweiz. III. Mitteilung: Gegenwärtige Arztbehandlung-Prävalenzstudie. Schweiz. med. Wschr. 109, 652 (1979).

(4) BIENER K., ROSCHEWSKI L., SCHAER M.: Normogrammstudie Frauen. Repräsentativstudie über das Gesundheitsprofil berufstätiger Frauen. Schweiz. med. Rundschau (Praxis) 12, 401 (1980).

Adresse der Autoren:

Institut für Sozial- und Präventivmedizin der Universität zürich, Gloriastrasse $32 \mathrm{~b}, \mathrm{CH}-8006$ zürich 